\title{
The expression of the tomato prosystemin in tobacco induces alterations irrespective of its functional domain
}

\author{
Giandomenico Corrado $^{1}$ - Simona Arena ${ }^{2}$ Tania Araujo-Burgos ${ }^{1} \cdot$

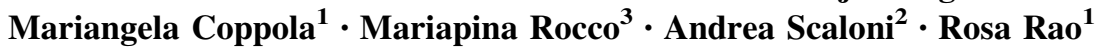

Received: 12 January 2016/Accepted: 20 February 2016/Published online: 1 March 2016

(c) The Author(s) 2016. This article is published with open access at Springerlink.com

\begin{abstract}
Systemin is a tomato peptide hormone that promotes plant defense against plant-chewing pests. This octadecapeptide is released from the C-terminal region of prosystemin, a 200 amino acid-long cytosolic precursor. Homologues of the tomato prosystemin are present in other Solanaceae but not in tobacco. This species does not respond to the exogenous application of the tomato systemin. Previously, it was shown that the expression of the full-length tomato prosystemin cDNA in tobacco affects the proteomic repertoire and increases plant tolerance against phytopathogenic fungi. In this work, to evaluate the relevance of the systemin sequence, we generated transgenic tobacco plants that express a mutated prosystemin lacking the systemin region. By using proteomics and gene expression analyses, we show that the constitutive expression of the truncated prosystemin altered the proteomic profile of tobacco leaves and increased plant resistance against $B$. cinerea. The overlap of the modifications caused by the expression of the full-length and the truncated prosystemin indicated that these alterations occur also in the absence of the systemin sequence. Although the cellular mechanisms of prosystemin cleavage are elusive, our work demonstrates
\end{abstract}

Electronic supplementary material The online version of this article (doi:10.1007/s11240-016-0967-8) contains supplementary material, which is available to authorized users.

Rosa Rao

rao@unina.it

1 Dipartimento di Agraria, Università degli Studi di Napoli Federico II, Via Università 100, 80055 Portici, NA, Italy

2 ISPAAM, National Research Council, Via Argine 1085, 80147 Naples, Italy

3 Dipartimento di Scienze e Tecnologie, Università degli Studi del Sannio, Via Port'Arsa 11, 82100 Benevento, Italy that the expression of a heterologous cytosolic peptidehormone precursor, irrespective of the presence of its peptide domain, associated with unpredicted changes at the proteomic and transcriptional level.

Keywords Nicotiana tabacum - Pathogen resistance . Proteomics · Prohormone

\section{Introduction}

Systemin is an octadecapeptide isolated from tomato leaves as inducer of the systemic defense against herbivores (Pearce et al. 1991). The main role of this peptide is to prime the octadecanoid pathway, which produces the plant hormone jasmonic acid and its derivatives. In tomato, systemin is synthesized as part of a larger precursor protein of 200 amino acids called prosystemin (Mcgurl et al. 1992; Mcgurl and Ryan 1992). The prosystemin contains four imperfect repeated regions of nine amino acids (37-45; $80-88 ; 117-125$ and $145-153)$ and a truncated 6 amino acid repeated motif at the $\mathrm{N}$-terminal region (3-8), while the systemin sequence is located in the $\mathrm{C}$-terminal region (amino acids 179-196) (Mcgurl and Ryan 1992). This structure has been considered suggestive of gene duplication/elongation events (Mcgurl and Ryan 1992). Later studies indicated that the presence and the arrangement of those repeats are shared among prosystemin sequences in both Solanaceae (potato, bell pepper and black nightshade) and other plants (prince's feather, beetroot and quinoa) (Pearce 2011). As systemin is the only known active peptide hormone released from its precursor, it is currently unknown whether prosystemin has any biological function other than being an intermediate in the synthesis of systemin (Dombrowski et al. 1999). 
The prosystemin lacks a signal peptide and accumulates in the cytosol. The conversion process from prohormone to hormone is currently unknown. It has been discussed that prosystemin is an inactive precursor of systemin, whose wound-inducible processing is a prerequisite for the systemin release and the amplification of a systemic wound signaling (Pearce 2011). The prosystemin gene is normally transcribed at low level. Wounding, as well as other stresses, increases strongly its expression (Matsubayashi and Sakagami 2006; Mcgurl et al. 1992). The constitutive overexpression of the prosystemin cDNA is associated with a constitutive accumulation of proteinase inhibitors as well as the generation of a systemic signal, implying a continuous release of the systemin peptide from its precursor (Mcgurl et al. 1994).

Homologs of the tomato systemin have been identified in members of the Solaneae subtribe but not in evolutionarily more distant species such as tobacco (Constabel et al. 1998). Biologically active peptides functionally closer to systemin were later isolated in tobacco and other Solanales (tomato, petunia, black nightshade and Ipomoea batatas) (Pearce 2011). Although these peptides are structurally unrelated to systemin, they were named hydroxyprolinerich systemins (HypSys) because of their systemin-like function (Pearce 2011). While systemin primary sequences are highly conserved between species, HypSys are more divergent and are all characterized by the presence of a proline or hydroxyproline-rich central domain. The HypSys precursors are secreted and processed into more than one active peptides, giving rise in tobacco to two, in petunia and tomato to three, and in sweet potato to probably six peptides (Chen et al. 2008; Pearce et al. 2001; Pearce and Ryan 2003; Pearce et al. 2007).

We previously showed that the expression of the fulllength tomato prosystemin in tobacco modified the plant proteome and affected plant resistance against fungal pathogens (Rocco et al. 2008). Tobacco does not respond to the exogenous application of the tomato systemin (Scheer et al. 2003) and it does not have a systemin homologue. In the present study, we investigated if the above-mentioned effects are influenced by the presence of the systemin peptide. To this goal, we analyzed transgenic tobacco plants that express a truncated form of the tomato prosystemin, which lacks the systemin region. We demonstrate that the constitutive expression of this truncated prosystemin form affected host protein accumulation and increased tobacco resistance against $B$. cinerea, indicating that these effects are not only due to the systemin sequence.

\section{Materials and methods}

\section{Plant genetic transformation}

The pPS binary vector (Rocco et al. 2008), containing the coding region of the prosystemin cDNA under the control of the CaMV 35S RNA promoter and the pea rbcS terminator, was digested with $B g l \mathrm{II}$ and $X b a$ I (Promega) to release a $71 \mathrm{bp}$ fragment that encompasses the region coding for the systemin peptide. The protruding ends were filled in by the large (Klenow) fragment of the DNA PolymeraseI (Promega). After a gel-based purification, the plasmid was self-ligated by the T4 DNA ligase (Promega). All the enzymatic reactions were performed according to the manufacturer's instructions. The resulting binary vector was named pPRO (Fig. 1). Agrobacterium tumefaciens LBA4404 cells were transformed by electroporation and used to produce transgenic tobacco (Nicotiana tabacum cv. Samsun NN) lines as described (Rocco et al. 2008). Kanamycin-resistant putative transformants were screened by PCR with the oligo pK-pro (5'-TATCCTTCGCAAGAC) and rbcs-rev (5'-GTGC GCAATGAAACAG3), which anneal to the CaMV35S promoter and the rbcs terminator, respectively. PCR positive transgenic lines were maintained in vitro under kanamycin selection $\left(100 \mathrm{mg} \mathrm{l}^{-1}\right)$ and the number of transgenic insertions was verified by a Southern blot analysis. Homozygous $T_{2}$ plants were obtained by single seed descent analysis of $\mathrm{T}_{1}$ plants with a single transgenic insertion. Four-six weeks old plants, grown in greenhouse conditions, were used for molecular analysis and bioassays.

\section{DNA isolation and manipulation}

Genomic DNA was isolated from leaves using the Gen Elute Plant Genomic DNA miniprep kit (Sigma-Aldrich). Southern blot analysis was carried out as described (Corrado et al. 2005) using the Hind III restriction enzyme (Promega) and as a probe a gel-purified PCR product obtained from the pPRO8 plasmid with the BBS forward and rbcs-rev primers (Supplementary Table 1). For PCR amplification, a $50 \mu \mathrm{lmix}$ was prepared containing $100 \mathrm{ng}$ of DNA template, $0.4 \mu \mathrm{M}$ primers, $1.5 \mathrm{mM} \mathrm{MgCl}_{2}, 100$ $\mu$ MdNTPs and $1 \mathrm{U}$ Taq DNA Polymerase (Promega) in $1 \times$ PCR buffer (Promega). The primer sequences and their temperature of annealing are reported in Supplementary Table 1. PCR reactions were performed in a Mastercycler Gradient (Eppendorf). 


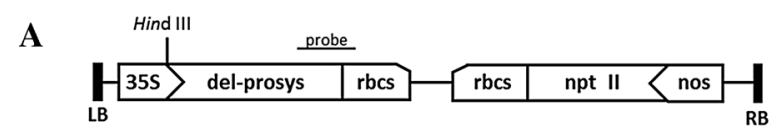

B

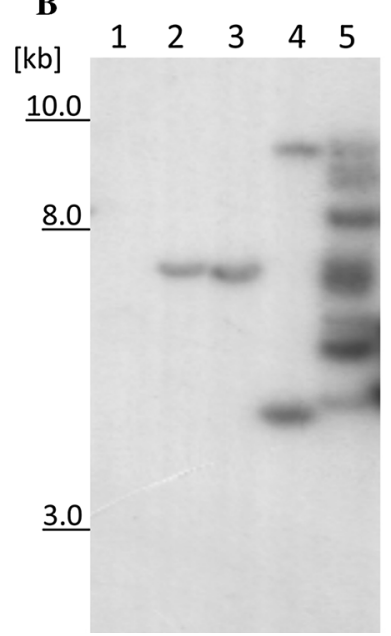

C
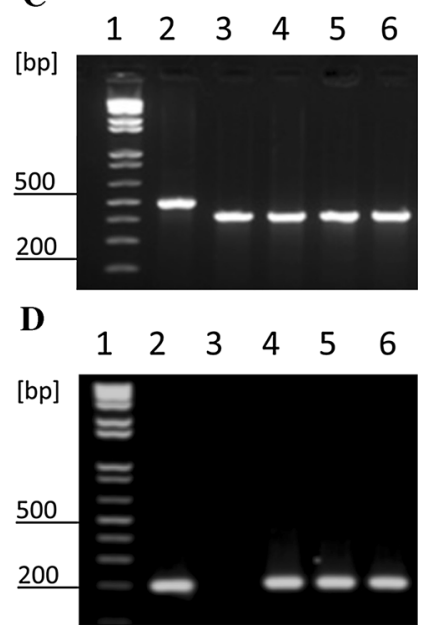

Fig. 1 Characterization of the transgenic plants. a T-DNA map of the pPRO8 binary vector used for plant genetic transformation showing the restriction site used for Southern blot analysis (not to scale). $L B$ : T-DNA left border sequence, 35S CaMV: 35S RNA gene promoter, del-prosys: deleted prosystemin cDNA sequence, $r b c S$ : polyA addition sequence of the pea rbcS gene, npt II: neomycin phosphotransferase II coding sequence, nos: nopaline synthase promoter, RB: T-DNA right border sequence. b Southern blot analysis. The position of the Hind III restriction site is indicated on the top of the map. The fragment probe employed is marked with a line. 1 N. tabacum "Samsun" NN, 2 PRO856, 3 PRO8-38, 4 PRO8-30, 5 PRO8-22. Numbers at the left margin indicate marker fragment sizes. c Amplification of the EF1- $\alpha$ retrotranscripts to control for the cDNA synthesis efficiency and to detect possible contamination of genomic DNA in the PCR reactions. Numbers at the left margin indicate marker fragment sizes. $11 \mathrm{~kb}$ plus DNA ladder (Invitrogen), $2 \mathrm{~N}$. tabacum genomic DNA, 3 "Samsun" NN cDNA, 4 MZ 119 cDNA, 5 PRO8-56 cDNA, 6 PRO838 cDNA. d Amplification of the prosystemin cDNA. Numbers at the left margin indicate marker fragment sizes. $11 \mathrm{~kb}$ plus DNA ladder (Invitrogen), 2 pPRO8 plasmid, 3 "Samsun" NN cDNA, 4 MZ 119 cDNA, 5 PRO8-56 cDNA, 6 PRO8-38 cDNA

\section{RNA isolation and RT-PCR}

Total RNA was extracted from leaves using a previously published procedure (Corrado et al. 2007). Single-strand cDNA synthesis and quantitative reverse transcription (RT)-PCR were also performed as described (Coppola et al. 2013). Primer sequences and their main features are reported in Supplementary Table 1. We analyzed three plants per line and reactions were carried out in triplicates. The $\mathrm{Ct}$ values were averaged for each triplicate. The efficiency of cDNA synthesis was checked by amplifying the elongation factor-1 alpha (EF-1 $\alpha$ ) cDNA with primers (NT-EF-fw and NT-EF-rev) designed to anneal on two consecutive exons to detect possible contaminant DNA (Corrado et al. 2005). Relative quantitation of gene expression was carried out using the $2^{-\Delta \Delta \mathrm{Ct}}$ method, using the EF- $1 \alpha$ as an endogenous reference gene for the normalization of the expression levels of the target genes. Relative fold change variations in expression levels are reported as $2^{-\Delta \Delta \mathrm{Ct}}$ values. The statistical significance of the results was evaluated as reported (Corrado et al. 2007).

\section{Protoplasts isolation}

Protoplasts were prepared from transgenic leaves (approx. $6 \mathrm{~g}$ ) as described (Tortiglione et al. 2003). Proteins in the protoplast medium were precipitated with $70 \% \mathrm{NH}_{4} \mathrm{SO}_{2}$ and suspended in $1 \times$ PBS buffer. Proteins within protoplasts were isolated in homogenization buffer $(200 \mathrm{mM}$ Tris-Cl, $300 \mathrm{mM} \mathrm{NaCl}, 1 \%$ Triton, $1 \mathrm{mM}$ EDTA, $2 \mathrm{mM}$ PMSF; $\mathrm{pH}$ 8.0). After sonication (30 s), cell debris was removed by centrifugation for $20 \mathrm{~min}$ at $12,000 \mathrm{~g}$ at $4{ }^{\circ} \mathrm{C}$.

\section{Proteomic analysis}

Protein extraction, Western blot, 2-D electrophoresis, image acquisition, image analysis, protein digestion, MS analysis and protein identification were performed as already described, since the proteomic analysis was carried out alongside with the analysis of the transgenic plants expressing the fulllength prosystemin precursor (Rocco et al. 2008).

\section{Functional annotation of identified proteins}

Functional annotation was carried out by sequence analysis using the Blast2GO software (Gotz et al. 2008). Briefly, a blastp similarity search (e-value $<1 \mathrm{e}-6$ ) against the $\mathrm{nr}$ NCBI protein database was performed to retrieve a maximum of 20 homologous hits per query. GO-term mapping and annotation were retrieved using NCBI as well as nonredundant reference protein databases (PSD, UniProt, Swiss-Prot, TrEMBL, RefSeq, GenPept, PDB Full Gene Ontology DB). Additional annotations (e.g. the recovery of implicit "Biological Process" and "Cellular Component" GO-terms from "Molecular Function" annotations) were implemented using ANNEX 5.0. Completion of the functional annotation with protein domain information was performed with Inter ProScan 5.0. A plant GO-Slim reduction was carried out to summarize the functional content of the dataset. On the basis of the richness of the different GO levels (from 1 to 11), we generated a level 3-hierarchical summary to present meaningful GO identifiers that are at hand yet not excessively general.

\section{Fungal bioassay}

Five-week old transgenic plants were tested for resistance to $B$. cinerea, a necrotrophic airborne pathogen that attacks fruit, stems, leaves and flowers of many plant species, 
causing typically a grey mold-disease. The assays were carried out as described (Di Maro et al. 2010) with the following modifications: four plants per line and two leaves per plant were analyzed; a volume of $10 \mu \mathrm{l}$ of spore suspension $\left(10^{6}\right.$ spores $\left./ \mathrm{ml}\right)$ was used for four inocula per leaves; lesions were measured at 4, 6 and 8 days after inoculation.

\section{Results}

\section{Generation and analysis of the transgenic plants}

Tobacco plants (N. tabacum "Samsun" NN) were stably transformed via Agrobacterium with a construct containing a prosystemin cDNA sequence lacking the $3^{\prime}$ region coding for the systemin peptide. In the binary vector employed, named pPRO8, the expression cassette consists of the deleted prosystemin cDNA under the control of the constitutive CaMV 35SRNA promoter and the pea rbcs terminator. A schematic representation of the T-DNA region of the pPRO8 plasmid is shown in Fig. 1a. Putative transformants, named PRO8, were screened by PCR (not shown) and Southern-blot hybridization to confirm the presence and the number of T-DNA insertions, respectively (Fig. 1b). Two single-insertion transgenic lines (PRO8-32 and PRO8-56) were selected for further investigations. Transgene expression was assayed by using an RT-PCR approach (Fig. 1c, d; Supplementary Fig. 1). In this analysis, we included also the transgenic tobacco line (MZ119) that expresses the full-length tomato prosystemin (Rocco et al. 2008). This line was obtained using a similar construct in an independent transformation experiment and in this work, it served as a positive control to evaluate the possible effects in the PRO8 lines. Transgenic lines did not display any obvious phenotypic abnormality compared to the untransformed tobacco plants (not shown).

\section{Subcellular localization of the modified prosystemin precursor}

In order to monitor the subcellular localization of the mutated prosystemin in tobacco, protoplasts isolated from leaves of the transgenic plants and their medium were analyzed by Western blot. The result showed an immunepositive signal for the protoplast extract, indicating that that the modified precursor is mainly synthesized and stored in the cytoplasm (Fig. 2). The protein had an apparent molecular weight of approximately $28 \mathrm{kDa}$. As previously reported, the mobility of the prosystemin protein in a standard SDS-PAGE is higher than its predicted mass $(23 \mathrm{kDa})$ most likely because of the high percentage (44\%) of charged amino acids (Delano et al. 1999).

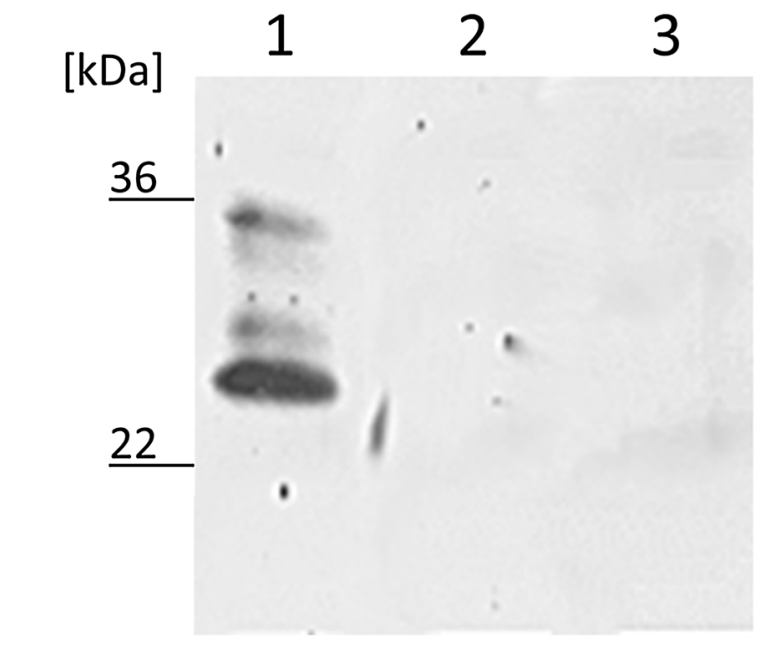

Fig. 2 Intracellular accumulation of the modified prosystemin in tobacco. Western blot analysis of the intracellular protein content from protoplasts (1) and of the secreted proteins in the incubation medium (2) of transgenic PRO8-56 leaves. 3 Total protein extract from leaves of untransformed plants. Numbers at the left margin indicate marker fragment sizes in $\mathrm{kDa}$

\section{Identification of differentially represented proteins}

We used a proteomic approach to monitor the molecular effects of the constitutive expression of the deleted prosystemin gene. Representative Coomassie-stained gels are reported in Fig. 3. Average proteomics maps showed 236 (control) and 253 (transgenic) spots, $89 \%$ of which were found to be present in both groups. Normalization and statistical analysis of quantitative proteomics data indicated that 24 spots were differentially represented (fold change $\geq 2$ or $\leq 0.5$ ) in leaves of transformed plants compared to controls $(p<0.05)$. For protein identification, these spots together with other 17 (selected as a reference for their constant abundance across samples) were excised from the gels, proteolyzed and subjected to MS analysis. Database search using data deriving from MALDI-TOF peptide mass fingerprinting (PMF) experiments allowed the identification of 23 spots, while the remaining ones $(n=18)$ were identified by $\mu$ LC-ESI-IT-MS/MS. The list of the identified proteins is reported in Table 1 along with their relative quantification. A poly-ubiquitin like protein showed the highest variation in quantitative terms.

Functional annotation of the differentially represented proteins and data mining on the resulting annotations, predominantly based on the gene ontology (GO) vocabulary, was performed by sequence similarity search. For the "biological process" domain, a broader overview of the ontology content was achieved by using the GO-plant slim list. The most abundant richness of the annotations was obtained at level 3 (Supplementary Fig. 2). Analysis of GO term associations for biological process at level 3 is 

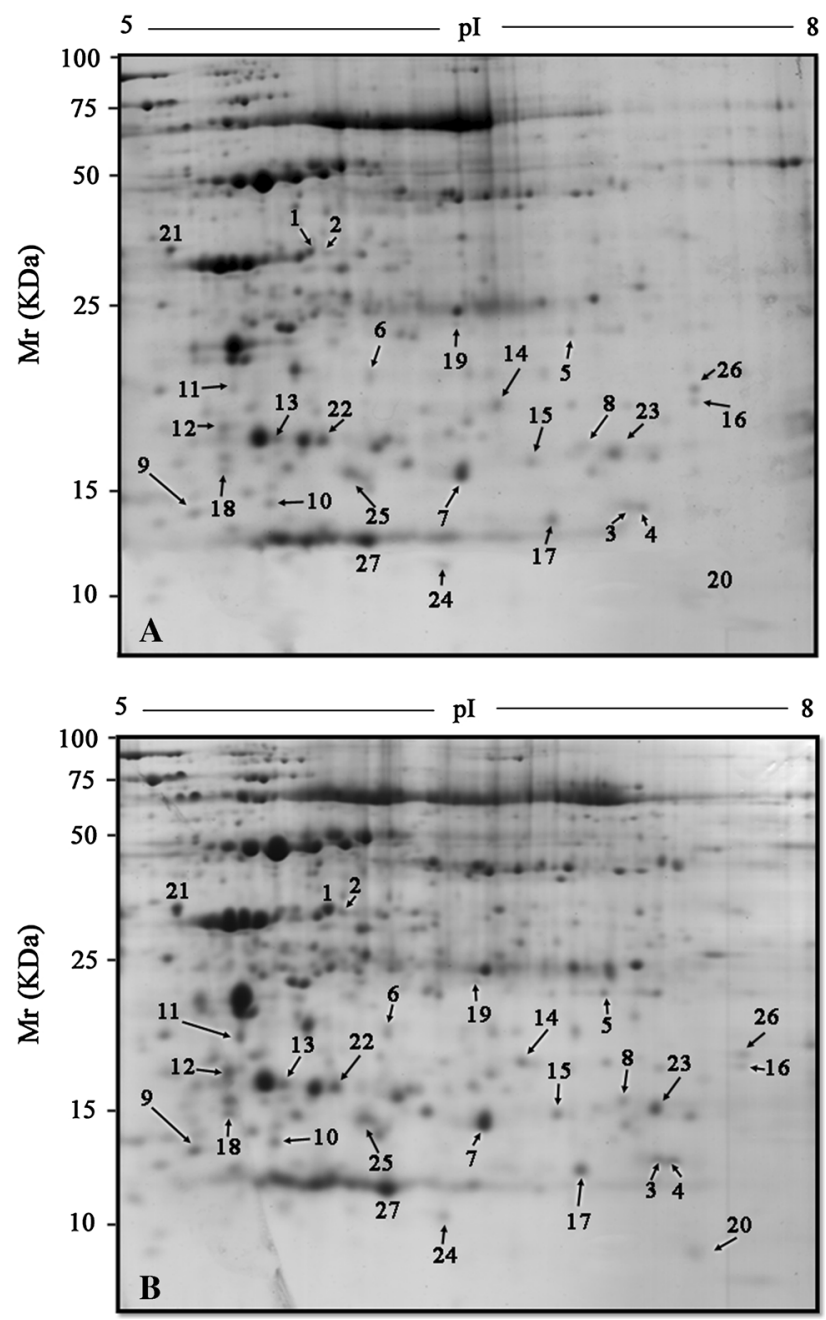

Fig. 3 2-DE proteomic maps of leaves from untransformed tobacco and transgenic plants expressing the deleted prosystemin precursor. Representative 2-DE gels of leaves from $N$. tabacum untranformed (a) and transgenic PRO8-56 (b) plants. Protein spots are numbered as in Table 1. Protein extracts were analyzed in first dimension $(\mathrm{pH} 5-8$ linear IPG, $18 \mathrm{~cm}$ ); second dimension was performed on a vertical slab gel $(12 \% \mathrm{~T})$

reported in Fig. 4. The "organic substance metabolic process", "primary metabolic process", "cellular metabolic process" and "response to stress" were the most represented series of molecular events or functions that, at the proteomic level, were affected by the expression of the deleted prosystemin. According to the mapping of the enzymatic activities of the differentially represented proteins, the KEGG-reference pathways "Photosynthesis", "Carbon Fixation in Photosynthetic Organisms" and "Oxidative phosphorylation" was the most affected.

With the aim to study the communality and specificity of the effects of the deleted and the full-length prosystemin, we compared the identified proteins of the PRO8 plants with the ones of the plants expressing the full-length prosystemin. 21 out of the 24 spots differentially represented in the PRO8 proteomic maps were also differentially represented in the MZ119 leaves, indicating a very high overlap of the effects of the two different heterologous proteins in the independently obtained transgenic lines.

\section{Evaluation of the resistance to fungal pathogens}

To evaluate if the expression of the deleted tomato prosystemin alters the tobacco response to necrotrophic fungi, leaves from 5-week old transgenic PRO8 lines and from untransformed plants were inoculated with $B$. cinerea spores. Lesions were measured at 4,6 and 8 days after inoculation. Disease severity was quantified by measuring the necrotic areas. Transgenic tobaccos showed smaller lesions than control plants (Fig. 5), implying that the expression of the deleted prosystemin increased pathogen tolerance in tobacco.

\section{Effects on endogenous gene expression}

The bioassays indicated that the expression of the deleted prosystemin increases the resistance against necrotrophic fungi. This result is consistent to what we already reported for the MZ transgenic plants, which express the full-length prosystemin protein (Rocco et al. 2008). For this reason, we evaluated the expression of genes associated to plant defense in both types of transgenic lines. We analyzed genes related to plant stress response, such as a heat-shock protein (HSP) (Park and Hong 1998), a stromal ascorbate peroxidase (SAP) (Yoshimura et al. 2002), a glutathione S-transferase (GST) (Takahashi and Nagata 1992) and the proteinase inhibitor II (Pin II) (Balandin et al. 1995). Furthermore, we investigated collectively the expression of the two endogenous tobacco prosystemin-like hydroxyproline-rich glycopeptides (TobHypSys I and II) (Pearce et al. 2001). Due to the high similarity of the coding sequences, primers were designed to amplify the transcripts of both genes. The results indicated that the GST, involved in oxidative stress and detoxification, and HSP, involved in response to environmental stress, are up-regulated in MZ119, in accordance with the proteomic study (Rocco et al. 2008), while SAP was not differentially expressed (Fig. 6). Furthermore, differences between MZ119 and the two PRO8 lines were present only for the GST gene. Pin II was overexpressed in both types of transgenic lines, although at a level that is much lower than in tomato plants overexpressing the prosystemin (Coppola et al. 2015). The relative quantification of the expression level of tobacco hydroxyproline-rich glycopeptides HypSys I and II showed an overexpression in both types of transgenic lines. Overall, the data indicated that the constitutive accumulation of heterologous 


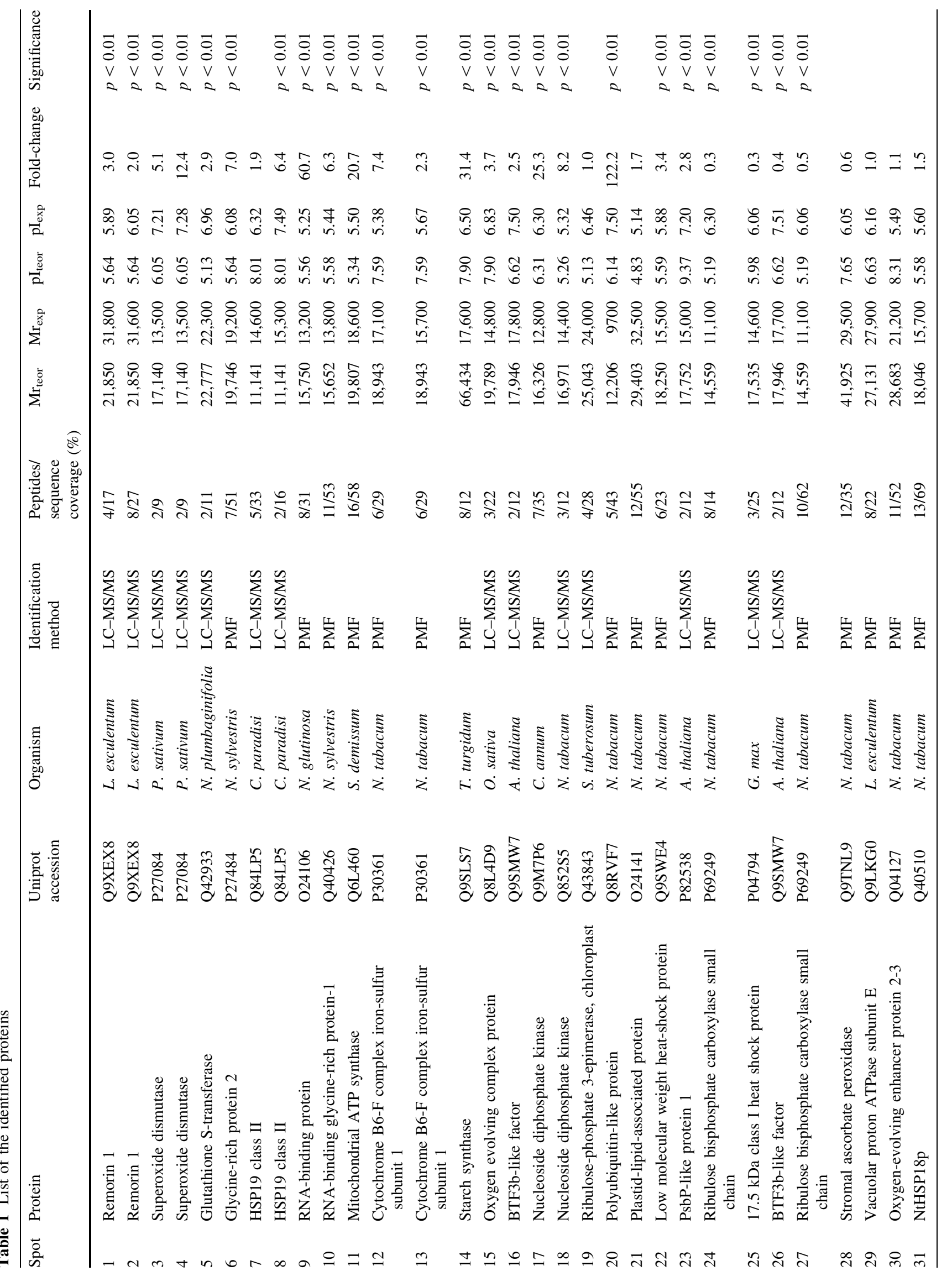




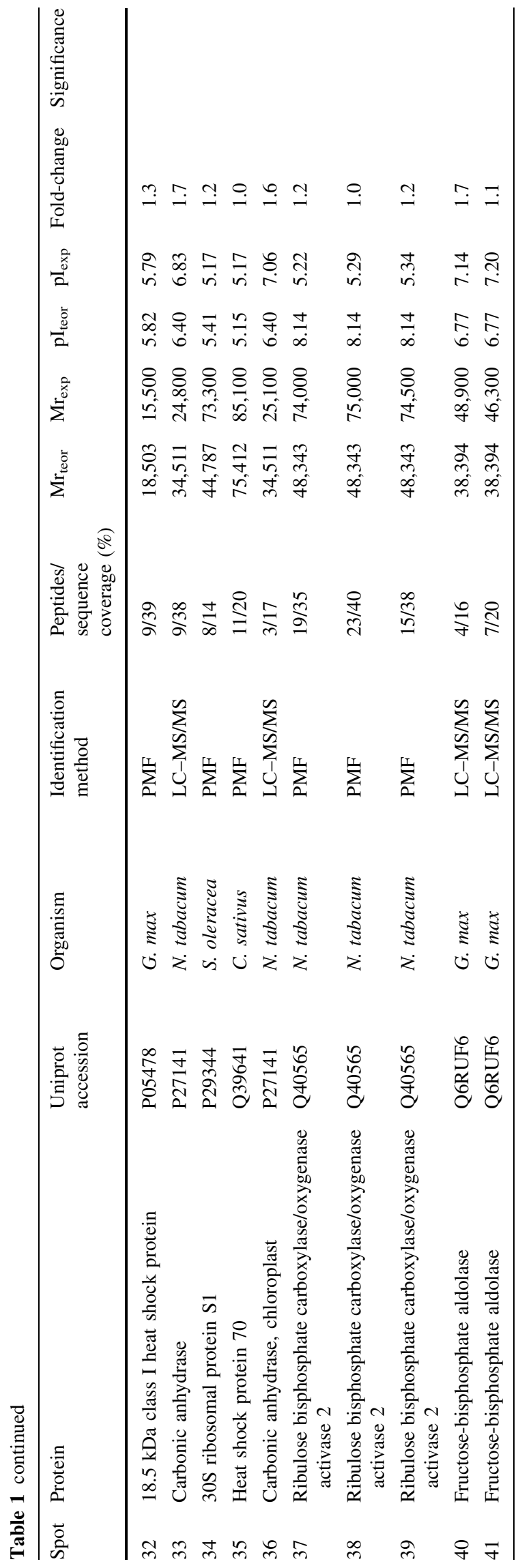

precursors of a signalling molecule, such as the tomato prosystemin and its mutated version, affected the expression of genes involved in plant response to stress.

To estimate the relevance of the observed variations, the expression level of HSP, GST, Pin II and TobHypSys was quantified in tobacco leaves of the "Samsun" NN control following mechanical wounding. The experiment was carried out with 4 week-old plants and leaves were harvested $6 \mathrm{~h}$ following the treatment. The relative quantification indicated that HSP, GST and Pin II were overexpressed after wounding (Fig. 7). The expression of the endogenous tobacco prosystemin-like HypSys was not different. This is consistent to previous evidence indicating that the expression of the HypSys gene in wounded leaves increases after $6 \mathrm{~h}$, reaching the maximum at $24 \mathrm{~h}$ (Ren et al. 2008). More interestingly, the RQ values following wounding were comparable to those of the transgenic lines. The only exception was represented by the GST gene, which was not differentially expressed in the PRO8 lines.

Overall, the data indicated that the expression of the tomato prosystemin in tobacco increases the transcription of some stress-related genes. Such effect is also present in transgenic plants that express a precursor lacking the region encoding the systemin peptide. At both proteomic and transcriptomic level, however, we observed differences between the two transgenic types, implying that the C-terminal region of the tomato prosystemin also affects the activation of gene expression in tobacco.

\section{Discussion}

Peptide signaling is crucial for various aspects of plant growth, development and stress response (Matsubayashi and Sakagami 2006). Plants respond also to peptides produced by biotic stressors (Albert 2013; Schwessinger and Ronald 2012). Furthermore, signaling peptides involved in plant response to biotic stress can also derive from intracellular proteins involved in other metabolic functions (Pearce et al. 2010; Schmelz et al. 2006). The majority of plant propeptides are synthesized through the secretory pathway but, bioactive peptides derive also from cytosolic precursors, as in the case of systemin (Yamaguchi and Huffaker 2011). A previous study indicated that the expression of the tomato systemin precursor in tobacco affects the host proteomic repertoire and influences the resistance against fungal pathogens (Rocco et al. 2008). Despite the similarities of the wound signaling between tomato and tobacco (Kandoth et al. 2007; Sudha and Ravishankar 2002; Yu et al. 2015), the latter does not have a prosystemin homologue. However, it is still a matter of debate whether tobacco cells respond to exogenously 
Fig. 4 Pie charts summary representation of GO-annotation results. Relative distribution of "Biological Process" terms following $\mathrm{GO}$ classification of the identified differentially expressed proteins (see Table 1) at level 3 (sequence cut-off association: $>5$ )
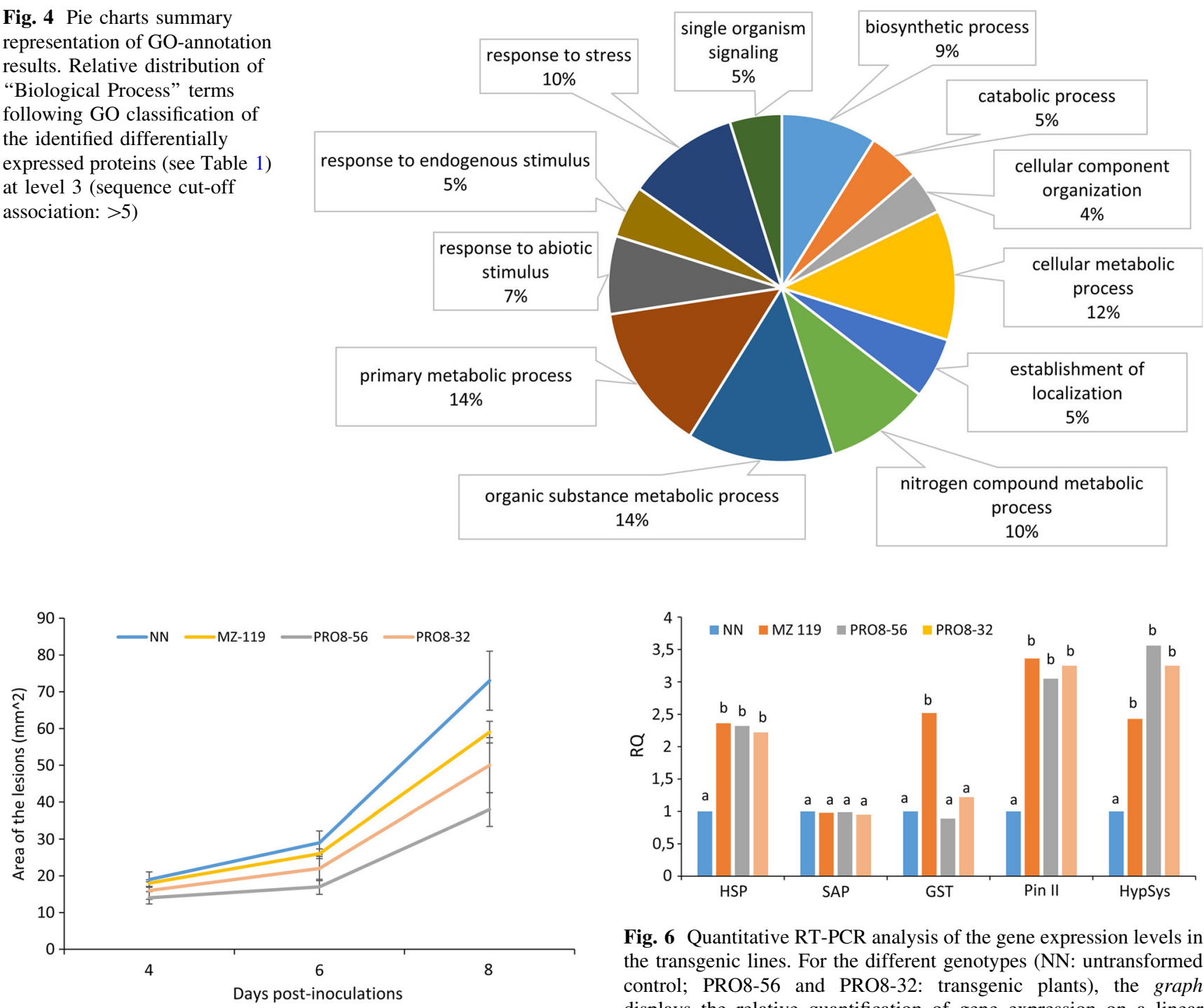

Fig. 5 Increased tolerance to Botrytis cinerea. Average lesion areas at 4, 6 and 8 days after inoculation (error bars report the standard error of the mean). NN: untransformed control; PRO8-56, PRO8-38 and MZ-119: transgenic plants. PRO8-56, PRO8-38 and MZ-119 plants were significantly different from the untransformed control at 8 days (Student $t$ test; $* * p<0.01$ )

applied systemin (Malinowski et al. 2009; Scheer et al. 2003).

In this work, we investigated whether a modified prosystemin protein that lacks the systemin peptide alters gene expression in tobacco. Proteomic analysis showed that several biological functions were affected by the expression of the deleted prosystemin. Pathways involving an organic substance and by which plant cells transform chemical substances were the most represented biological functions in our dataset of differentially represented proteins. In addition, the functional annotations of the identified proteins were related also to stress response. The deleted prosystemin increased the accumulation of some

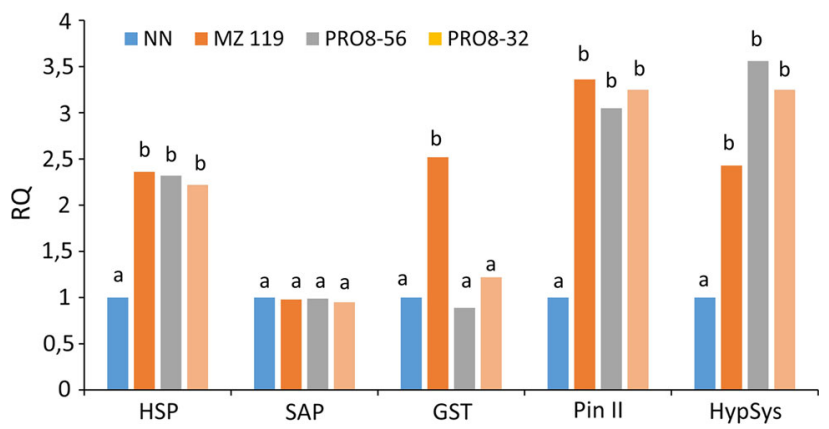

Fig. 6 Quantitative RT-PCR analysis of the gene expression levels in the transgenic lines. For the different genotypes (NN: untransformed control; PRO8-56 and PRO8-32: transgenic plants), the graph displays the relative quantification of gene expression on a linear scale (RQ). RQs are shown relative to the calibrator NN genotype. For each gene, different letters indicate groups with different statistical significance, based on the ANOVA analysis of the $2^{-\Delta \mathrm{Ct}}$ values (Tukey; $\alpha=0.05$ ) the oxidative cellular stress, as it occurred in the tobacco plants expressing the full-length prosystemin (Rocco et al. 2008). In accordance with previous results, the gene expression analysis confirmed that the prosystemins upregulate some stress-related genes. In addition, the present work indicated that the effects in tobacco involve also other classes of genes. Transgenic plants showed a very moderate up-regulation of the protease inhibitor II (Pin II) gene, when compared to tomato (Mcgurl et al. 1994). Furthermore, the expression of the native and mutated prosystemin precursors affected the endogenous tobacco HypSys genes. In tomato, systemin and Hyp-rich glycopeptides cooperatively activate defense-related genes (Pearce et al. 2001). Taking into account that this effect is independent from the members of the cellular system involved in the control of 


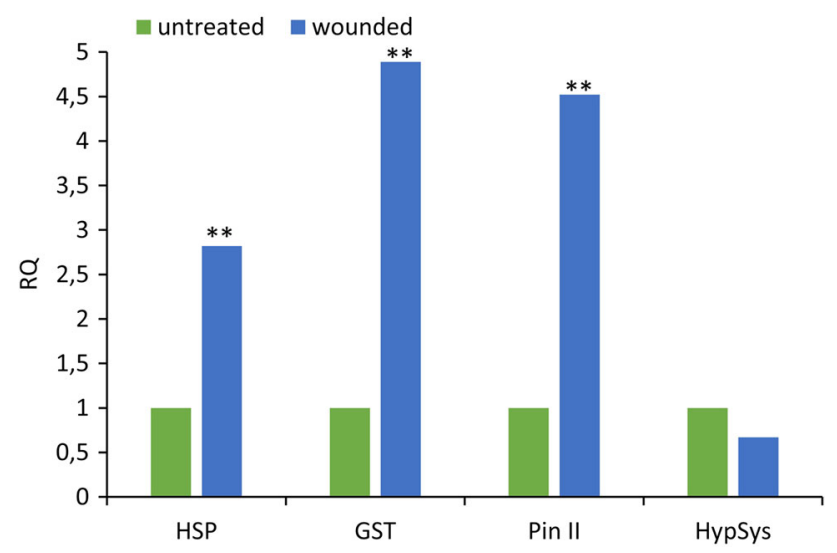

Fig. 7 Quantitative RT-PCR analysis of the gene expression levels in leaves following wounding. The graph displays the relative quantity (RQ) on a linear scale of tobacco genes $6 \mathrm{~h}$ following mechanical wounding. RQs are shown relative to the calibrator control condition (untreated). For each gene, asterisks indicate that the $2^{-\Delta \mathrm{Ct}}$ values were significantly different between the control and the test conditions (Student $t$ test; **p $<0.01$ )

presence of the systemin domain, it is more likely that the tobacco Hypsys activation is a direct or indirect response to the effects of the activation of stress-related genes.

Since prosystemin processing in tomato is currently unknown, it is difficult to speculate whether the prosystemin directly activates some defense pathways in tobacco or the observed effects relate to its proteolytic products (Albert 2013). In tomato, the exogenous supply of prosystemin induces similar responses as systemin (Dombrowski et al. 1999; Vetsch et al. 2000) and the deletion of systemin abolishes this effect (Vetsch et al. 2000). These data are also consistent with the unexpected evidence that prosystemin-dependent activation of defense gene expression does not require wounding or any other stress (Mcgurl et al. 1994). This study indicated that systemin signaling is regulated via the biosynthesis of prosystemin rather than processing, controlled secretion or both. In tomato, the extracytosolic proteolytic activity is not sufficient to produce detectable levels of systemin (Dombrowski et al. 1999), suggesting that intracellular proteases are responsible for the generation of the systemin peptide. Our study indicated that also the modified prosystemin is not secreted and predominantly accumulates in the cytoplasm. Previously, it has been shown that the TMOF peptide, engineered to replace the systemin in the tomato prosystemin, is detectable in transgenic tobacco plants that express this chimeric precursor (Tortiglione et al. 2003), supporting the occurrence of proteolytic events in tobacco. However, the possible presence of other products released from the multiple processing of the precursor has never been investigated. For all these reasons, we speculate that the prosystemin protein, for its intrinsic features [e.g. multibasic recognition sites exhibiting motifs such as the $(\mathrm{K} / \mathrm{R}) \mathrm{X}_{\mathrm{n}}(\mathrm{K} / \mathrm{R})$ ] undergoes "default" degradation by intracellular proteases (Asher et al. 2006), as it occurs in mammals for a number of prohormones (Bhattacharyya et al. 2014; Scamuffa et al. 2006). A likely candidate system for the formation of processed peptides is the ubiquitin/proteasome-dependent processing (RUP) system (Bhattacharyya et al. 2014; Scamuffa et al. 2006; Verma et al. 2001). The effect of both types of prosystemin types can be explained assuming that these precursors go through multiple processing events in tobacco, which may lead to a direct or indirect activation of oxidative stress defense mechanisms (Albert 2013; Pearce et al. 2010; Schmelz et al. 2006). In potato, the expression of a modified heterologous antimicrobial peptide precursor provided resistance against plant pathogens but, unexpectedly, altered also the plant ROS response to stress (Goyal et al. 2013). These data open the intriguing possibility that plant cells sense the constitutive expression of heterologous prohormones as a chronic perturbation of protein homeostasis, activating some molecular stress responses (Morimoto 2008).

In conclusion, our work demonstrated that the proteomic and transcriptional alterations as well as the increased plant tolerance to phytopathogenic fungi observed in the transgenic tobaccos are not related exclusively to the presence of the systemin domain, indicating that the constitutive accumulation of a heterologous precursor of a signaling molecule can have unpredicted effects. Even though tobacco lacks a prosystemin homologue, this complex alteration has some common elements with the output observed typically in plant response to stress, such as mechanical wounding. Although further work is necessary to unravel cellular mechanisms of the prohormone conversion, our study also provides new insights about the prosystemin processing.

Acknowledgments This work was partially supported by the GENOPOMPRO Project (Cod. PON02_00395_3082360).

Authors' contributions GC contributed to the work plan, supervised the experimental work, analyzed the data and wrote the paper, SA performed the proteomic work, TAB performed bioassays and the molecular work, MC provided assistance to the bioinformatics analysis, MP carried out the 2DE experiments, AS contributed to the work plan, supervised the experimental work and analyzed the proteomic data, RR conceived the work, supervised the experimental work and contributed to the work plan.

Open Access This article is distributed under the terms of the Creative Commons Attribution 4.0 International License (http://crea tivecommons.org/licenses/by/4.0/), which permits unrestricted use, distribution, and reproduction in any medium, provided you give appropriate credit to the original author(s) and the source, provide a link to the Creative Commons license, and indicate if changes were made. 


\section{References}

Albert M (2013) Peptides as triggers of plant defence. J Exp Bot 64(17):5269-5279. doi:10.1093/Jxb/Ert275

Asher G, Reuven N, Shaul Y (2006) 20S proteasomes and protein degradation "by default". BioEssays 28(8):844-849. doi:10. 1002/Bies.20447

Balandin T, van der Does C, Albert JM, Bol JF, Linthorst HJ (1995) Structure and induction pattern of a novel proteinase inhibitor class II gene of tobacco. Plant Mol Biol 27(6):1197-1204

Bhattacharyya S, Yu H, Mim C, Matouschek A (2014) Regulated protein turnover: snapshots of the proteasome in action. Nat Rev Mol Cell Biol 15(2):122-133. doi:10.1038/nrm3741

Chen YC, Siems WF, Pearce G, Ryan CA (2008) Six peptide wound signals derived from a single precursor protein in Ipomoea batatas leaves activate the expression of the defense gene sporamin. J Biol Chem 283(17):11469-11476

Constabel CP, Yip L, Ryan CA (1998) Prosystemin from potato, black nightshade, and bell pepper: primary structure and biological activity of predicted systemin polypeptides. Plant Mol Biol 36(1):55-62

Coppola V, Coppola M, Rocco M, Digilio MC, D'Ambrosio C, Renzone G, Martinelli R, Scaloni A, Pennacchio F, Rao R, Corrado G (2013) Transcriptomic and proteomic analysis of a compatible tomato-aphid interaction reveals a predominant salicylic acid-dependent plant response. BMC Genomics 14:515. doi:10.1186/1471-2164-14-515

Coppola M, Corrado G, Coppola V, Cascone P, Martinelli R, Digilio MC, Pennacchio F, Rao R (2015) Prosystemin overexpression in tomato enhances resistance to different biotic stresses by activating genes of multiple signaling pathways. Plant Mol Biol Report 33(5):1270-1285. doi:10.1007/s11105-014-0834-x

Corrado G, Bovi PD, Ciliento R, Gaudio L, Di Maro A, Aceto S, Lorito M, Rao R (2005) Inducible expression of a Phytolacca heterotepala ribosome-inactivating protein leads to enhanced resistance against major fungal pathogens in tobacco. Phytopathology 95(2):206-215

Corrado G, Sasso R, Pasquariello M, Iodice L, Carretta A, Cascone P, Ariati L, Digilio MC, Guerrieri E, Rao R (2007) Systemin regulates both systemic and volatile signaling in tomato plants. J Chem Ecol 33(4):669-681

Delano JP, Dombrowski JE, Ryan CA (1999) The expression of tomato prosystemin in Escherichia coli: a structural challenge. Protein Expr Purif 17(1):74-82. doi:10.1006/prep.1999.1113

Di Maro A, Terracciano I, Sticco L, Fiandra L, Ruocco M, Corrado G, Parente A, Rao R (2010) Purification and characterization of a viral chitinase active against plant pathogens and herbivores from transgenic tobacco. J Biotechnol 147(1):1-6. doi:10.1016/j. jbiotec.2010.03.005

Dombrowski JE, Pearce G, Ryan CA (1999) Proteinase inhibitorinducing activity of the prohormone prosystemin resides exclusively in the C-terminal systemin domain. Proc Natl Acad Sci USA 96(22):12947-12952. doi:10.1073/pnas.96.22.12947

Gotz S, Garcia-Gomez JM, Terol J, Williams TD, Nagaraj SH, Nueda MJ, Robles M, Talon M, Dopazo J, Conesa A (2008) Highthroughput functional annotation and data mining with the Blast2GO suite. Nucleic Acids Res 36(10):3420-3435. doi:10. 1093/Nar/Gkn176

Goyal RK, Hancock REW, Mattoo AK, Misra S (2013) Expression of an engineered heterologous antimicrobial peptide in potato alters plant development and mitigates normal abiotic and biotic responses. Plos One 8(10). doi:10.1371/journal.pone.0077505

Kandoth PK, Ranf S, Pancholi SS, Jayanty S, Walla MD, Miller W, Howe GA, Lincoln DE, Stratmann JW (2007) Tomato MAPKs LeMPK1, LeMPK2, and LeMPK3 function in the systemin- mediated defense response against herbivorous insects. Proc Natl Acad Sci USA 104(29):12205-12210. doi:10.1073/pnas. 0700344104

Malinowski R, Higgins R, Luo Y, Piper L, Nazir A, Bajwa VS, Clouse SD, Thompson PR, Stratmann JW (2009) The tomato brassinosteroid receptor BRI1 increases binding of systemin to tobacco plasma membranes, but is not involved in systemin signaling. Plant Mol Biol 70(5):603-616. doi:10.1007/s11103009-9494-X

Matsubayashi Y, Sakagami Y (2006) Peptide hormones in plants. Annu Rev Plant Biol 57:649-674. doi:10.1146/annurev.arplant. 56.032604.144204

Mcgurl B, Ryan CA (1992) The organization of the prosystemin gene. Plant Mol Biol 20(3):405-409. doi:10.1007/Bf00040600

Mcgurl B, Pearce G, Orozcocardenas M, Ryan CA (1992) Structure, expression, and antisense inhibition of the systemin precursor gene. Science 255(5051):1570-1573. doi:10.1126/science.1549783

Mcgurl B, Orozcocardenas M, Pearce G, Ryan CA (1994) Overexpression of the prosystemin gene in transgenic tomato plants generates a systemic signal that constitutively induces proteinase-inhibitor synthesis. Proc Natl Acad Sci USA 91(21):9799-9802. doi:10.1073/pnas.91.21.9799

Morimoto RI (2008) Proteotoxic stress and inducible chaperone networks in neurodegenerative disease and aging. Gene Dev 22(11):1427-1438

Park SM, Hong CB (1998) Comparison of the structure and expression pattern for a low molecular weight heat-shock protein cDNA clone from Nicotiana tabacum. Mol Cells 8(5):594-599

Pearce G (2011) Systemin, hydroxyproline-rich systemin and the induction of protease inhibitors. Curr Protein Pept Sci 12(5):399-408

Pearce G, Ryan CA (2003) Systemic signaling in tomato plants for defense against herbivores-isolation and characterization of three novel defense-signaling glycopeptide hormones coded in a single precursor gene. J Biol Chem 278(32):30044-30050. doi:10.1074/jbc.M304159200

Pearce G, Strydom D, Johnson S, Ryan CA (1991) A polypeptide from tomato leaves induces wound-inducible proteinase-inhibitor proteins. Science 253(5022):895-898. doi:10.1126/ science. 253.5022 .895

Pearce G, Moura DS, Stratmann J, Ryan CA (2001) Production of multiple plant hormones from a single polyprotein precursor. Nature 411(6839):817-820. doi:10.1038/35081107

Pearce G, Siems WF, Bhattacharya R, Chen YC, Ryan CA (2007) Three hydroxyproline-rich glycopeptides derived from a single petunia polyprotein precursor activate defensin I, a pathogen defense response gene. J Biol Chem 282(24):17777-17784. doi:10.1074/jbc.M701543200

Pearce G, Yamaguchi Y, Barona G, Ryan CA (2010) A subtilisin-like protein from soybean contains an embedded, cryptic signal that activates defense-related genes. Proc Natl Acad Sci USA 107(33):14921-14925. doi:10.1073/pnas.1007568107

Ren F, Lian HJ, Chen L (2008) TobpreproHypSys-A gene expression and defense protein activity in the tobacco wounding response. J Plant Biol 51(1):48-51

Rocco M, Corrado G, Arena S, D'Ambrosio C, Tortiglione C, Sellaroli S, Marra M, Rao R, Scaloni A (2008) The expression of tomato prosystemin gene in tobacco plants highly affects host proteomic repertoire. J Proteomics 71(2):176-185

Scamuffa N, Calvo F, Chretien M, Seidah NG, Khatib AM (2006) Proprotein convertases: lessons from knockouts. FASEB J 20(12):1954-1963. doi:10.1096/fj.05-5491 rev

Scheer JM, Pearce G, Ryan CA (2003) Generation of systemin signaling in tobacco by transformation with the tomato systemin receptor kinase gene. Proc Natl Acad Sci USA 100(17):10114-10117. doi:10.1073/pnas.1432910100 
Schmelz EA, Carroll MJ, LeClere S, Phipps SM, Meredith J, Chourey PS, Alborn HT, Teal PEA (2006) Fragments of ATP synthase mediate plant perception of insect attack. Proc Natl Acad Sci USA 103(23):8894-8899

Schwessinger B, Ronald PC (2012) Plant innate immunity: perception of conserved microbial signatures. Annu Rev Plant Biol 63(63):451-482

Sudha G, Ravishankar GA (2002) Involvement and interaction of various signaling compounds on the plant metabolic events during defense response, resistance to stress factors, formation of secondary metabolites and their molecular aspects. Plant Cell Tissue Organ Cult 71(3):181-212. doi:10.1023/A: 1020336626361

Takahashi Y, Nagata T (1992) parB: an auxin-regulated gene encoding glutathione S-transferase. Proc Natl Acad Sci USA 89(1):56-59

Tortiglione C, Fogliano V, Ferracane R, Fanti P, Pennacchio F, Monti LM, Rao R (2003) An insect peptide engineered into the tomato prosystemin gene is released in transgenic tobacco plants and exerts biological activity. Plant Mol Biol 53(6):891-902. doi:10. 1023/B:Plan.0000023667.62501.Ef
Verma R, McDonald H, Yates JR, Deshaies RJ (2001) Selective degradation of ubiquitinated Sic1 by purified $26 \mathrm{~S}$ proteasome yields active $S$ phase cyclin-Cdk. Mol Cell 8(2):439-448. doi:10. 1016/S1097-2765(01)00308-2

Vetsch M, Janzik I, Schaller A (2000) Characterization of prosystemin expressed in the baculovirus/insect cell system reveals biological activity of the systemin precursor. Planta 211(1):91-97. doi:10.1007/s004250000264

Yamaguchi Y, Huffaker A (2011) Endogenous peptide elicitors in higher plants. Curr Opin Plant Biol 14(4):351-357. doi:10.1016/ j.pbi.2011.05.001

Yoshimura K, Yabuta Y, Ishikawa T, Shigeoka S (2002) Identification of a cis element for tissue-specific alternative splicing of chloroplast ascorbate peroxidase pre-mRNA in higher plants. J Biol Chem 277(43):40623-40632. doi:10.1074/jbc. M201531200

Yu L, Yan J, Yang YJ, Zhu WM (2015) Overexpression of tomato mitogen-activated protein kinase SIMPK3 in tobacco increases tolerance to low temperature stress. Plant Cell Tissue Organ Cult 121(1):21-34. doi:10.1007/s11240-014-0675-1 